\title{
Jejunojejunal Intussusception after Feeding Jejunostomy: Rare Complication of a Common Surgery
}

\author{
Gaurav Maheshwari ${ }^{1}$ and Namrata Maheshwari ${ }^{2}$ \\ ${ }^{1}$ Department of Surgery, Kasturba Medical College, Manipal, Karnatka, India \\ ${ }^{2}$ Department of Anesthesiology, Kasturba Medical College, Manipal, Karnatka, India
}

Correspondence should be addressed to: Gaurav Maheshwari; drgauravmaheshwari@gmail.com

Received Date: 4 February 2014; Accepted Date: 16 April 2014; Published Date: 13 May 2014

Academic Editor: Ashok K V Kumar

Copyright (C) 2014 Gaurav Maheshwari and Namrata Maheshwari. Distributed under Creative Commons CC-BY 3.0

\begin{abstract}
Feeding jejunostomy is a very frequently performed procedure both for pre-operative feeding in carcinoma esophagus and also for early postoperative enteral feeding in major gastrointestinal surgeries. Feeding catheter induced jejunojejunal intussusceptions is an infrequent complication of feeding jejunostomy. We present a 34 year old female with carcinoma of upper third of esophagus with complete luminal obstruction who had jejunojejunal intussusception after feeding jejunostomy. Though, uncommon these complications should be reported in literature, as proactively managing these rare complications or preventing them will significantly improve outcomes in patients with feeding jejunostomy.
\end{abstract}

Keywords: Jejunojejunal intussusception, Feeding jejunostomy.

\section{Introduction}

An intussusception is an invagination of a segment of the gastrointestinal tract into an adjacent one. Jejunojejunal intussusception is a rare complication of placing of the jejunostomy tube. Only case reports have been published about jejunojejunal intussusception following feeding jejunostomy needing relaparotomy. This report emphasizes that even a simple surgical procedure like feeding jejunostomy should be followed up as close as any other major surgical procedure, because these rare complication can significantly increases morbidity once in a while.

\section{Case Report}

A 34 year old female with diagnosed squamous cell carcinoma of upper third of esophagus was referred to surgical department for feeding jejunostomy. After adequate counseling and clearance, Witzel's type of feeding jejunostomy was performed. She was tolerating jejunostomy feeds well till the fourth post operative day, when she had upper abdominal discomfort and colicky pain. Her clinical examination revealed minimal upper abdominal fullness. She was managed with prokinetics and antacids, but her symptoms aggravated in form of severe abdominal pain, upper abdomen distension and increase in pain.

Cite this Article as: Gaurav Maheshwari and Namrata Maheshwari (2014), "Jejunojejunal Intussusception after Feeding Jejunostomy: Rare Complication of a Common Surgery," JMED Research, Vol. 2014 (2014), Article ID 483746, DOI: 10.5171/2014.483746 
Feeds were withheld, and her radiological tests revealed features suggestive of small bowel obstruction. Ultrasound was not informative in view of excess bowel gas. Due to financial reasons and emergent nature of the pathology, CT scan was deferred and she was taken up for laparotomy.

Jejunojejunal intussusception without gangrenous changes was detected (Fig 1) and jejunostomy tube was placed in normal position. There was a significant closed loop like obstruction in the presence of completely obstructed tumour in esophagus with gross dilatation of stomach.
She was successfully treated by operative reduction of intussusception without resection of the small intestine. She had an uneventful recovery post operatively and was discharged from the hospital on $8^{\text {th }}$ post operative day. The presence of complete obstruction justified laparotomy. In our patient, the lead point of intussusception might not be feeding jejunostomy site as intra-operatively, the intussusception was few inches distal to the expected feeding jejunostomy site. Post-operative adhesions might have served as an extra luminal factor and a pathological lead point for the jejunojejunal intussusception.

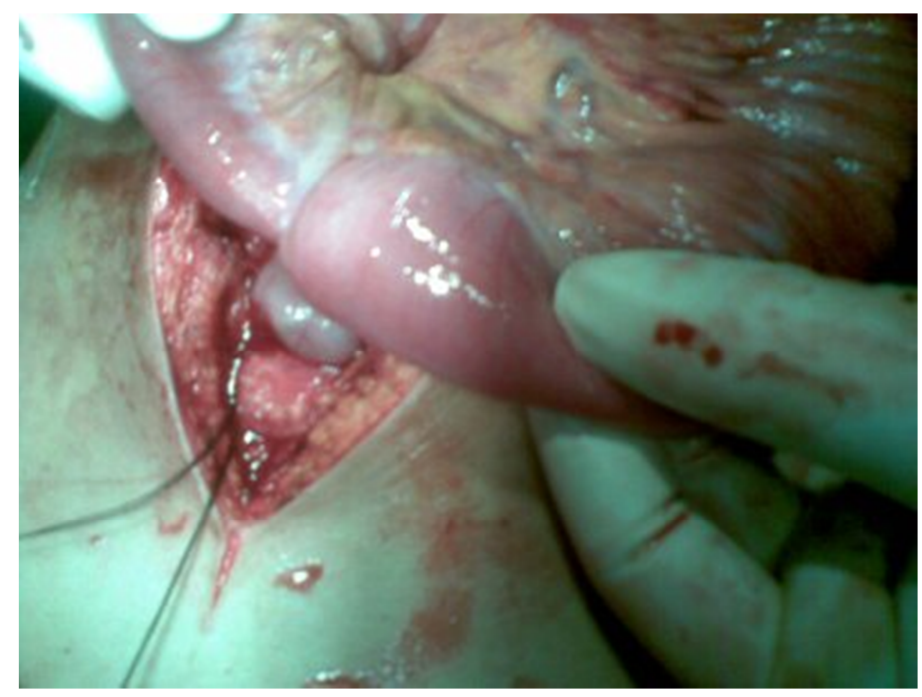

Fig. 1: Intraoperative Jejunojejunal Intussusception without Gangrenous Change

\section{Discussion}

Intussusception, the telescoping of a segment of bowel loop into another is one of the leading causes of intestinal obstruction in children. But it is rare in adults, accounting for $<1 \%$ of all cases of small bowel obstruction and $5 \%$ of all cases of intussusception. Intussusception has been classified into four groups' mainly tumor-related, post-operative, miscellaneous, idiopathic ${ }^{1}$. As per review of literature, common complications associated with feeding jejunostomy are mechanical due to tube displacement, obstruction or migration, infectious which can be subcutaneous or intraabdominal2, gastrointestinal symptoms like nausea, vomiting, diarrhea, and abdominal distension $^{3}$, and metabolic abnormalities ${ }^{4}$ like hyperglycemia, hypokalemia, hypophosphatemia, and hypomagnesaemia.) ${ }^{5-7}$. $\quad$ Feeding jejunostomy as a cause for jejunojejunal intussusception has been reported rarely ${ }^{8}$.

In a study by Carucci et al. ${ }^{9}$ complications were reported in 40 (14\%) out of 280 cases at radiographic examination in patients. These complications included small-bowel obstruction, small-bowel strictures, and intraabdominal collections. Other rare complications reported were jejunal hematomas and intussusception of the small bowel $(1 / \%)$ at the site of the jejunostomy tube. Of four patients who had 
small-bowel intussusceptions, enteral feeding was continued without difficulty in three patients ${ }^{9}$. Only in one case, intussusception was associated with smallbowel obstruction. This suggested that intussusception caused by jejunostomy tube may not interfere with tube feeding. Resection of the bowel segment of intussusception to prevent recurrence is not necessary ${ }^{10}$.

Review of literature suggested that the mechanism of jejunojejunal intussusception was likely to be due to a retrograde peristalsis of jejunum during vomiting episodes, or an injecting force produced by the tube feeding with pump infusion on the jejunostomy tube which acts as a stent. Besides, most of these patients requiring tube feedings are with lean body mass with small amount of fatty tissues (omentum, mesentery) in the wide abdominal cavity. This might allow the small bowel loops to move extensively in a free space, and may precipitate the intestine to undergo intussusception. Reymond et al suggested that adherence of two bowel segments with either an intraluminal (polyp) or extraluminal (postoperative adhesion) leadpoint can lead to intussusception ${ }^{11}$. But the exact etiology and mechanism has still not been described.

\section{Conclusion}

1. Jejunojejunal intussusception is a very rare complication of feeding jejunostomy which needs vigilant clinical suspicion in a patient of persistent high obstructive GI symptoms.

2. UGI series may not be diagnostic, so abdominal sonography or CT should be arranged if the jejunojejunal intussusception is highly suspected. Although the radiographic finding of intussusception may be incidental and transient, the patient might have a long segment intussusception and may not resolve itself.

3. This rare complication can be managed by operative reduction only, and resection is not advised if there is no gangrene change, perforation nor stenosis. It may not be necessary to take down the jejunostomy.

4. Reporting of rare complications for a common surgical procedure like feeding jejunostomy will certainly increases awareness among the surgeons, and thus improving the outcome of the patients.

\section{References}

1. Agha, F. P. (1986). "Intussusception in Adults," American Journal of Roentgenology. 146:527-31.

2. Cataldi-Betcher, E. L., Seltzer, M. H., Slocum, B. A. et al. (1983). "Complications Occurring During Enteral Nutrition Support: A Prospective Study," JPEN. Journal of Parenteral and Enteral Nutrition, 7:546-552.

3. Cogen, R., Weinryb, J., Pomerantz, C. \& Fenstemacher, P. (1991). "Complications of Jejunostomy Tube Feedings in Nursing Facility Patients," The American Journal of Gastroenterology, 86: 1610-1613.

4. Whiteley, G. S., Baildam, A. D., Walter, D. P. et al. (1992). "Complications of Percutaneous Endoscopic Enterostomy Tubes," Surgical Laparoscopy \& Endoscopy, 2:227-229.

5. Shike, M., Latkany, L., Gerdes, H. \& Bloch, A. S. (1996). "Direct Percutaneous Endoscopic Jejunostomies for Enteral Feeding," Gastrointestinal Endoscopy, 44:536540.

6. Eddy, V. A., Snell, J. E. \& Morris, J. A. (1996). "Analysis of Complications and Long Term Outcome of Trauma Patients with Needle Catheter Jejunostomy," The American Surgeon, 62:40-44.

7. Prahlow, J. A. \& Barnard, J. J. (1998). "Jejunostomy Tube Failure: 
Malnutrition Caused by Intraluminal Antegrade Jejunostomy Tube Migration," Archives of Physical Medicine and Rehabilitation, 79: 453455.

8. Zahurhussain, M. A., Bhat, A. G. Ahangar, A. M. Dar, G. N. Lone, Reyaz, et al. (2006). "Unusual Complications Offeeding Jejunostomy, Feeding Catheter Induced Jejuno-Jejunal Intussusceptions,' Jkpractitioner, 13:103.

9. Carucci, L. R., Levine, M. S., Rubesin, S. E., Laufer, I., Assad, S. \& Herlinger, H. (2002). "Evaluation of Patients with Jejunostomy Tubes: Imaging Findings," Radiology, 223:241-247.
10. Wu, T. H., Lin, C. W. \& Yin, W. Y. (2006). "Jejunojejunal Intussusception Following Jejunostomy," Journal of the Formosan Medical Association, 105 (4): 355-358.

11. Reymond, R. D. (1972). "The Mechanism of Intussusceptions: A Theoretical Analysis of the Phenomenon," The British Journal of Radiology, 45:1-7. 